\title{
On determining the cover of a simplex by spheres centered at its vertices
}

\author{
L. G. Casado • I. García • B. G. Tóth · E. M. T. Hendrix
}

Received: 12 January 2009 / Accepted: 6 January 2010 / Published online: 23 January 2010

(C) The Author(s) 2010. This article is published with open access at Springerlink.com

\begin{abstract}
The aim of this work is to study the Simplex Cover (SC) problem, which is to determine whether a given simplex is covered by spheres centered at its vertices. We show that the SC problem is equivalent to a global optimization problem. We investigate its characteristics.
\end{abstract}

Keywords Covering $\cdot$ Simplex $\cdot$ Spheres

\section{Introduction}

This study is motivated by earlier work on testing whether simplices representing the design area of blending problems may contain feasible solutions. The spheres represent areas where certainly no feasible solution can be located. This means, that if a simplex is covered, then

\begin{abstract}
This work has been funded by grants from the Spanish Ministry of Science and Innovation (TIN2008-01117), Junta de Andalucía (P06-TIC-01426, P08-TIC-3518), in part financed by the European Regional Development Fund (ERDF). Eligius Hendrix is a fellow of the Spanish "Ramon y Cajal" contract program, co-financed by the European Social Fund. B.G.-Tóth was supported by the János Bolyai fellowship of the Hungarian Academy of Sciences.
\end{abstract}

L. G. Casado · I. García $(\varangle)$

Computer Architecture and Electronics Department, University of Almería, 04120 Almeria, Spain e-mail: igarcia@ual.es

L. G. Casado

e-mail: leo@ual.es

B. G. Tóth

Department of Differential Equations,

Budapest University of Technology and Economics, Budapest, Hungary

e-mail: bog@math.bme.hu

E. M. T. Hendrix

Department of Computer Architecture, University of Málaga, Campus de Teatinos, 29017 Málaga, Spain e-mail: Eligius.Hendrix@wur.nl 
the simplex can be excluded from the search, because it certainly does not contain a feasible solution [2,5]. This paper studies the characteristics of the SC problem. The reasoning is illustrated with graphical examples and validated by several lemmas and a theorem.

We formulate the problem and its ingredients. A simplex is a set of convex combinations of $n$ affine independent vertices $v_{1}, \ldots, v_{n}$,

$$
S=\left\{x=\sum_{j=1}^{n} \lambda_{j} v_{j}, \sum_{j=1}^{n} \lambda_{j}=1 ; \lambda_{j} \in[0,1]\right\}
$$

Usually the vertices are defined in Euclidean space where the dimension $d$ should at least be $n-1$. Regular simplices are special cases where all the edges have the same length. The next ingredient of the SC problem are the spheres or balls with radius $r_{i}$ around the vertices,

$$
B_{i}=\left\{x \in R^{d}:\left\|x-v_{i}\right\| \leq r_{i}\right\}
$$

In our investigation we are specifically interested in the Euclidean distance norm $\|.\|_{2}$. Now the Simplex Cover problem can be formulated.

Simplex cover (SC) problem: Given an instance of a simplex $S$ and a set of spheres $B_{1}, \ldots, B_{n}$ around its vertices. Certify if

$$
\forall x \in S, \quad x \in \cup_{i} B_{i}
$$

or alternatively, check whether

$$
\exists x \in S, \quad x \notin \cup_{i} B_{i} .
$$

An equivalent representation of the SC problem can be used that focuses far more on distance from the vertices. This comes closer to the concept of Laguerre Voronoi diagrams (or Power diagrams) that is elaborated in Sect. 2. We focus on squared Euclidean distance. Define the function

$$
\varphi(x)=\min _{i}\left\{\left\|x-v_{i}\right\|^{2}-r_{i}^{2}\right\}
$$

where the squared distance is additively weighted [1]. Notice that function $\varphi$ being a minimum of strict convex functions is not convex. Moreover, in the specific case where none of the spheres $B_{i}$ is covering one of the other vertices $v_{j}, i \neq j, \varphi$ has local minima of $-r_{i}^{2}$ in each vertex; $\varphi\left(v_{i}\right)=-r_{i}^{2}$. The SC problem is equivalent to

$$
\forall x \in S \varphi(x) \leq 0 .
$$

Examples of the $\varphi$ function can be seen in Figs. 1 and 2 .

Notice that for a regular instance of $S$, if one of the $B_{i}$ covers another vertex, it covers them all and SC is solved.

Fig. 1 Instance of SC problem with two vertices, where $S$ is not covered

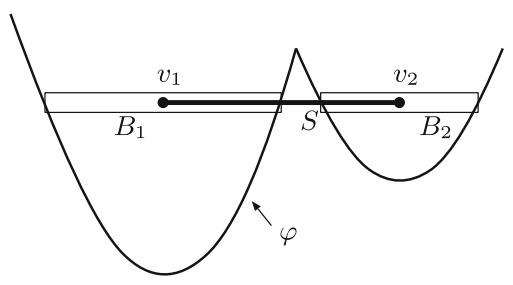


Fig. 2 Instance of SC problem with two vertices, where $S$ is covered

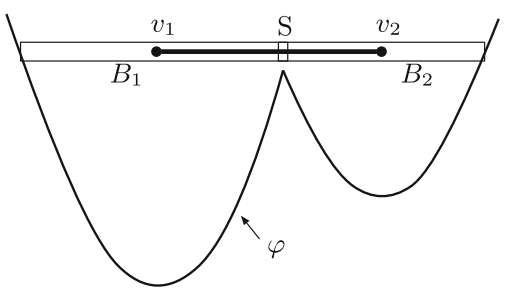

Equivalence (6) shows that it is interesting to study problem SCO (Simplex Cover Optimization):

$$
\Phi:=\max _{x \in S} \varphi(x)
$$

The SC problem has a certificate if $\Phi \leq 0$.

A lot of research is available on so-called Power sets $[3,6]$ that relates to $\varphi$ and mainly focuses on lower dimensional analysis. Our research deals with the general dimensional case. We elaborate this in Sect. 2. Problem SCO is not necessarily easier to solve than SC (3) or (4). One of the most obvious observations on the SC problem is that the simplex $S$ is covered if one of the spheres $B_{j}$ covers it. If this is not the case, we require properties on the SC problem of covering and the SCO problem of maximising $\varphi$ over $S$. In Sects. 3 and 4, we derive mathematical properties of the SC and SCO problems that can be used to solve them, which we illustrate by numerical examples. Specifically, Sect. 4.1 analyses the case of regular simplices, which is easier to solve and useful for the blending problem. Finally Sect. 5 summarises the found results.

\section{Distances, Voronoi diagrams and simplices}

Voronoi diagrams for a set of points $p_{i}$, divide the space into regions $D\left(p_{i}\right)$ where all points in $D\left(p_{i}\right)$ are closer to $p_{i}$ than to $p_{j}, j \neq i$. A survey of Voronoi diagrams can be found in [1]. There are several distance functions that can be used with respect to Voronoi diagrams. The usual is to consider the euclidean distance:

$$
d_{e}\left(p_{1}, p_{2}\right)=\left\|p_{1}-p_{2}\right\|_{2} .
$$

A Voronoi cell of $p_{i}$ for Euclidean distance are those points closest to $p_{i}$;

$$
D\left(p_{i}\right)=\left\{x \in R^{d} \mid d_{e}\left(x, p_{i}\right) \leq d_{e}\left(x, p_{j}\right), \quad j \neq i, j=1, \ldots, n\right\} .
$$

Each sphere $B_{i}$ in the SC problem has a radius $r_{i}$. This radius can be considered as a weight. Two ways to weight the distance are commonly considered and could be used for what are called weighted points, $p_{i}$ :

- Weight distance additively:

$$
d_{a}\left(x, p_{i}\right)=d_{e}\left(x, p_{i}\right)-r_{i}
$$

- Weight the so-called power distance:

$$
d_{p}\left(x, p_{i}\right)=d_{e}\left(x, p_{i}\right)^{2}-r_{i}^{2}
$$


(a)

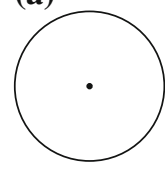

(b)

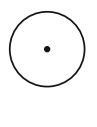

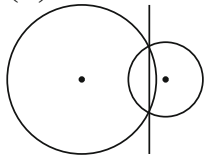

(c)

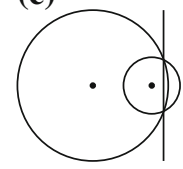

(d)

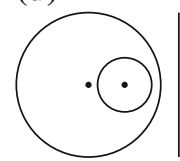

Fig. 3 Power planes of two weighted points on 4 different instances

Using $d_{a}$ in the Voronoi concept leads to diagrams with edges that are hyperbolic curves. Also so-called multiplicative Voronoi diagrams exist that have hyperbolic curves [1].

Life is a bit easier using $d_{p}$; the separating shapes are planes. One obtains a power diagram with power planes

$$
\Pi_{i j}=\left\{x \in R^{d} \mid d_{p}\left(x, p_{i}\right)=d_{p}\left(x, p_{j}\right)\right\}
$$

and power cells

$$
D_{p}\left(p_{i}\right)=\left\{x \in R^{d} \mid d_{p}\left(x, p_{i}\right) \leq d_{p}\left(x, p_{j}\right), \quad j \neq i j=1, \ldots, n\right\} .
$$

For a point $x$ outside sphere $B_{i}, d_{p}\left(x, p_{i}\right)$ is positive and $\sqrt{d_{p}\left(x, p_{i}\right)}$ represents the distance from the sphere with radius $r_{i}$ around $v_{i}$ to the point $x$ outside the sphere along the tangent line through the point $x$ [4]. The power Voronoi diagram (or power diagram) cells are convex polyhedra. Figure 3 shows the power diagram edges produced by two weighted points. The difference between a Euclidean Voronoi diagram and a Power diagram is that in the latter a cell can be empty, i.e., there is not a point inside (see Fig. 3d).

An important property of weighted and unweighted Voronoi diagrams is:

Property 1 Given $n+1$ affinely independent points with $n>1$. The Voronoi diagram contains one vertex of degree $n+1$ (number of edges incident to the vertex).

This has been shown for low dimensional cases in [1]. In Sect. 3 we will focus on the power diagram for the general dimensional case and derive a procedure to determine the corresponding so-called $\theta$ point. We will show that it plays a major role in the solution of SC and SCO problems.

\section{Covering a simplex, analysis based on the $\theta$-point}

The function

$$
\varphi(x)=\min _{i}\left\{d_{p}\left(x, v_{i}\right)\right\},
$$

is piecewise convex in each power cell. This implies that a maximum of $\varphi$ over a power cell is attained in one of its extreme points. It means that looking for the maximum value, $\Phi$ (see Eq.(7)), we can focus on the power planes $\Pi_{i j}$ that constitute the edges of power diagrams. Let us denote the interior of a set by int. Lemma 1 tells us that the solution of SCO should be looked for on the boundary of the power cells.

Lemma 1 A point $x^{*} \in \operatorname{argmax}_{x \in S} \varphi(x) \notin$ int $S \cap \cup_{i}$ int $D_{p}\left(v_{i}\right)$.

Proof For an interior point $x$ of a power cell around $v_{i}$,

$$
\varphi(x)=d_{p}\left(x, v_{i}\right)<d_{p}\left(x, v_{j}\right), \quad \forall j \neq i .
$$


This means that one can increase the value of $\varphi$ by going in the direction $\nabla \varphi(x)=2\left(x-v_{i}\right)$. Therefore $x$ cannot be a maximum point.

It is easy to check whether one sphere covers the complete simplex. In this case the maximum of $\varphi(x)$ may be attained at one or several vertices $v_{i}$ of $S$ (see Figs. 3c, d). For the case the maximum is not in a vertex, the lemma shows us that we can concentrate on the power planes $\Pi_{i j}$. Actually, it gives us the information that if the intersection of the power planes with the simplex are covered, i.e. $\varphi \leq 0$ on $S \cap \cup_{i j} \Pi_{i j}$, then all the simplex is covered. To illustrate these observations, we consider the line between the centers of the spheres of Fig. 3 as a simplex. One can see that in Fig. 3a the spheres do not intersect and the simplex is not covered in contrast to Fig. $3 \mathrm{~b}$. Notice that the intersection point of power plane and simplex corresponds to the global maximum point $x^{*}$. Figure $3 \mathrm{c}$ illustrates the case where one of the spheres covers the complete simplex and in Fig. 3d also the other sphere. Notice that in the last two cases, the power plane does not intersect with the simplex and the maximum point $x^{*}$ is found in a vertex of $S$.

We now focus on the situation where the maximum is attained at an interior point of $S$; i.e. $x^{*}=V \lambda$, where matrix $V=\left[v_{1}, v_{2}, \ldots, v_{n}\right]$ and $\lambda_{i}>0, \sum_{i} \lambda_{i}=1$.

Lemma 2 Consider problem SCO on a simplex with vertices $v_{1}, v_{2}, \ldots, v_{n}$ with corresponding $B_{i}$. Let $x^{*}=V \lambda$ be an interior maximum point of SCO.

$$
d_{p}\left(x^{*}, v_{1}\right)=d_{p}\left(x^{*}, v_{i}\right), \quad i=2, \ldots, n
$$

i.e. the power function of each vertex has the same value in $x^{*}$.

Proof Let the index set $I$ of active vertices be defined by $d_{p}\left(x^{*}, v_{i}\right)=\varphi\left(x^{*}\right)$ for $i \in I$. Assume that the lemma is not true: $x^{*}$ is interior maximum point and $\exists j, d_{p}\left(x^{*}, v_{j}\right)>\varphi\left(x^{*}\right)$ or $j \notin I$. In that case, one can construct a direction $u$ which is the projection of $v_{j}-x^{*}$ on the linear space where the active set remains active; $d_{p}\left(x^{*}+u, v_{i}\right)=\varphi\left(x^{*}+u\right)$. Direction $u$ is an ascent direction of $\varphi$ in the point $x^{*}$ and feasible, because $x^{*}$ is interior. This is a contradiction with $x^{*}$ being a maximum point.

The so-called $\theta$ point satisfying equation (15) fulfills the necessary condition for an interior optimum. Notice that $\theta$ can also be located outside $S$. The recipe to compute the $\theta$ point equating planes is derived from setting the power function values equal:

$$
\left(\theta-v_{1}\right)^{T}\left(\theta-v_{1}\right)-r_{1}^{2}=\left(\theta-v_{i}\right)^{T}\left(\theta-v_{i}\right)-r_{i}^{2}, \quad i=2, \ldots, n .
$$

Elaboration and bringing the terms with $\theta$ to the left hand side gives

$$
2\left(v_{i}-v_{1}\right)^{T} \theta=r_{1}^{2}-r_{i}^{2}+v_{i}^{T} v_{i}-v_{1}^{T} v_{1}, \quad i=2, \ldots, n .
$$

Equation (17) describes the plane separating $v_{1}$ and $v_{i}$ on equal power function value. The edge $\left(v_{i}-v_{1}\right)$ is perpendicular to the plane and the right hand side of the equation describes a constant. Focusing on a solution $\theta$, one can read (17) as linear equation $\left(v_{i}-v_{1}\right)^{T} \theta=$ constant. As point $\theta$ has to be in all the planes (17) for $i=2, \ldots, n$, we have $n-1$ linear equalities in either $\theta=V \lambda$ or in $\lambda$ which is $n$-dimensional.

$$
2\left(v_{i}-v_{1}\right)^{T} V \lambda=r_{1}^{2}-r_{i}^{2}+v_{i}^{T} v_{i}-v_{1}^{T} v_{1}, \quad i=2, \ldots, n .
$$

We are interested in the intersection with the plane through the points $v_{1}, v_{2}, \ldots, v_{n}$. It means that $\theta$ should move in that plane, such that the last linear equality is given by

$$
(1,1, \ldots, 1)^{T} \lambda=1 .
$$


Fig. 4 Graphical illustration of Example 1

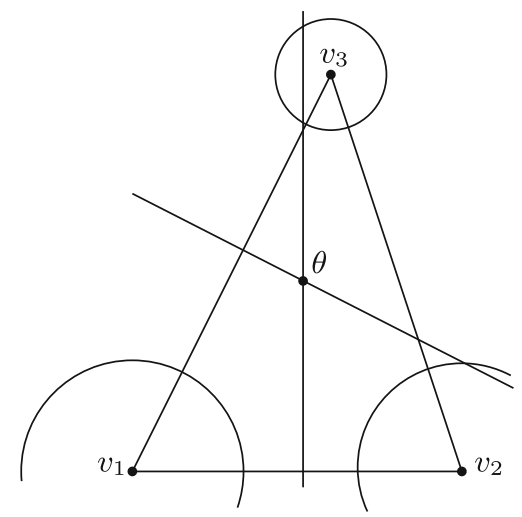

This means that finding $\theta$ can be done by solving a set of $n$ linear equalities. Given an interior $\theta$, we have the unique maximum point of SCO and one can simply check $d_{e}\left(\theta, v_{1}\right)^{2} \leq r_{1}^{2}$ to verify the solution of SC.

Example 1 Consider the following instance of three spheres in 2-dimensional space (see Fig. 4):

$$
v_{1}=\left(\begin{array}{l}
0 \\
0
\end{array}\right), v_{2}=\left(\begin{array}{l}
5 \\
0
\end{array}\right), v_{3}=\left(\begin{array}{l}
3 \\
6
\end{array}\right), r_{1}^{2}=4, r_{2}^{2}=3, r_{3}^{2}=1 \text {. }
$$

Point $\theta=\left(\begin{array}{l}2.6 \\ 2.7\end{array}\right)$ can be determined equating the two power planes: $\Pi_{12}$ between $v_{1}$ and $v_{2}$ and $\Pi_{13}$ between $v_{1}$ and $v_{3}$. The corresponding solution $\lambda=(0.3,0.25,0.45)^{T}$ is in the interior of the corresponding simplex. The point is on equal power function value $\varphi(\theta)=10.05$ for the three vertices.

Notice that if $\theta$ is covered by one of the spheres, it is also covered by all of them; $\theta \in B_{j}$ gives $\theta \in \cap B_{j}$. This is related to an earlier found result that says that if $\exists x \in S$ covered by all the spheres then the simplex is covered. It means that if $\theta$ is covered by one of the spheres then the simplex is fully covered. The result as proven in [2], is the following. Let conv denote the convex hull of a set.

Lemma 3 Given polytope $S=\operatorname{conv}\left\{v_{1}, \ldots, v_{h}\right\}$, with $v_{1}, \ldots, v_{h}$ the extreme points of $S$ (vertices) with corresponding spheres $B_{j}, j=1, \ldots$, h. If $\exists y \in \cap_{j} B_{j} \cap S$ then $S \subset \cup_{j} B_{j}$.

Figure 5 shows illustrative examples of 2-simplices. In Fig. 5a $S$ is not covered because the $\theta$ point is not covered. Figure 5b illustrates a simplex which is covered.

The next question is what to do if the computed $\theta$ is not an interior point of $S$; i.e. $\exists j \lambda_{j} \leq 0$, at least one of the $\lambda_{j}$ is not positive. It means that the maximum point $x^{*}$ of SCO is found at the boundary of $S$. We will investigate this further in Sect. 4.

Focusing on $\theta$, after finding $\theta$, it may be outside $S, \theta \notin S$, but covered by all spheres, $\theta \in \cap_{j} B_{j}$. The following lemma combined with Lemma 3, shows that this is also a sufficient condition to certify the covering of $S$ and no further analysis is required. It means that we do not need to solve problem SCO.

Lemma 4 Given a point $x=V \lambda, x \notin S$ and $x \in \cap_{j} B_{j}$. Then $\exists y \in \cap_{j} B_{j} \cap S$. 
(a)

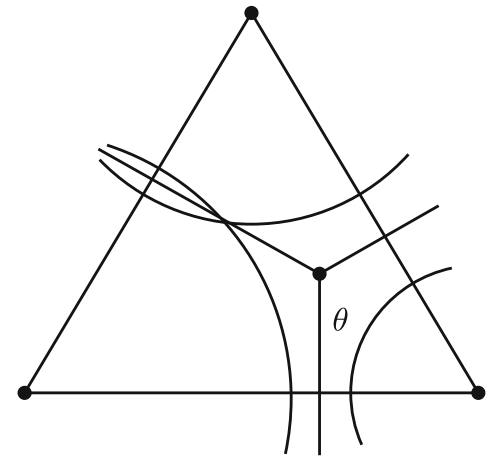

(b)

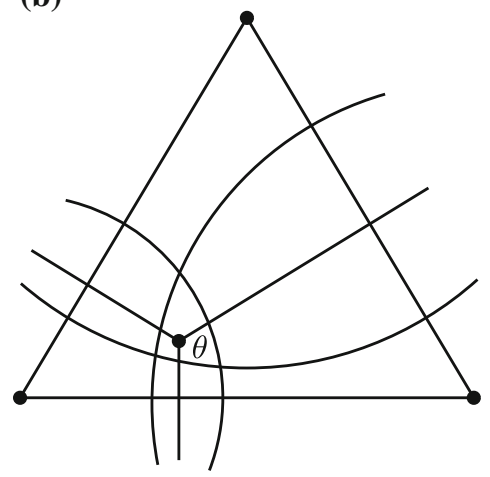

Fig. 5 Two instances of SC with corresponding $\theta$ point

Proof Without loss of generality, let $\lambda_{1}, \ldots, \lambda_{m} \geq 0$ and $\lambda_{m+1}, \ldots, \lambda_{n}<0$, so that $v_{m+1}, \ldots, v_{n}$ are at the other side of the facet where $x$ is closest to. We now make an orthogonal projection of $\left(y-v_{1}\right)=P\left(x-v_{1}\right)$ on the closest facet. One can do so by constructing an $n \times(m-1)$ matrix $X$ with elements $v_{j}-v_{1}$ for $j=2, \ldots, m$. An important observation is that in the construction of $X$ for the starting vector $v_{1}$, one can take any $v_{i}, i=1, \ldots, m$. The projection $y=v_{1}+X\left(X^{T} X\right)^{-1} X^{T}\left(x-v_{1}\right)$ results into $y \in S$. Notice that $\left\|y-v_{i}\right\|^{2}=\left\|P\left(x-v_{i}\right)\right\|^{2} \leq\left\|x-v_{i}\right\|^{2}, i=1, \ldots, m$ and $y$ is closer to $v_{j}, j=m+1, \ldots, n$. So $\left(y-v_{j}\right)^{T}\left(y-v_{j}\right) \leq\left(x-v_{j}\right)^{T}\left(x-v_{j}\right) \forall j$ such that $y \in \cap B_{j}$.

A consequence of combining Lemmas $1-4$, is the following theorem:

Theorem 1 Let $\theta$ be a solution of (18) and (19). If $\varphi(\theta) \leq 0$ then $S$ is covered.

Notice that this theorem is valid even for $\theta \notin S$. However, if $\theta \notin S$ and $\varphi(\theta)>0$, we have to investigate the boundary solutions of SCO in a more detailed way. As shown in the next section, also there the $\theta$-point plays a role.

\section{SCO has a boundary optimum}

If the maximum of $\varphi$ is not interior in $S$, it can be found on its boundary. In an extreme case, the maximum can be in a vertex of $S$ if one big sphere covers all the simplex $S$. Alternatively, maximum point $x^{*}$ can be attained at an edge, a triangular set or even higher dimensional face $C$ of $S$. Again the $\theta$-point plays a big role in the determination of face $C$. Consider the set $T=\operatorname{conv}\left\{v_{1}, \ldots, v_{n}, \theta\right\}$. Now one can see that

$$
\theta=\underset{x \in T}{\operatorname{argmax}} \varphi(x)
$$

because for all $x \in T, \varphi$ increases in the direction of $\theta$ such that $\theta$ is the global maximum point of (20). Moreover, $\theta$ is the common vertex of the power diagram. As $S \subset T$ also $\varphi(\theta) \geq \Phi$. That reconfirms the argumentation that a negative $\varphi(\theta)$ tells us that $S$ is covered.

If $\varphi(\theta)$ is positive, we are still interested in solving SCO. Figure 6 illustrates a simplex for which the $\theta$ point is located outside. The corresponding values of $\lambda_{1}$ and $\lambda_{2}$ are positive and $\lambda_{3}$ has a negative value. $S$ is covered because the intersection of $S$ with the power planes are covered. 
Fig. 6 Instance of SC with corresponding $\theta$ point not covered and outside of $S$

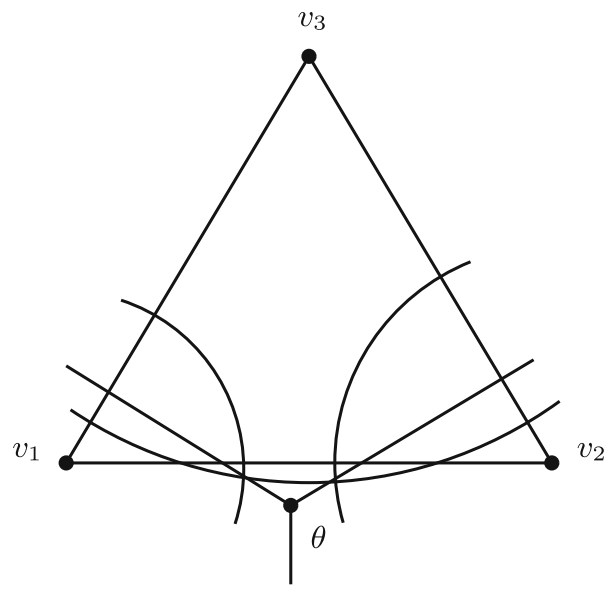

The previous analysis also helps us to determine the binding facet $C$ of $S$ where maximum points $x^{*}$ with $\Phi=\varphi\left(x^{*}\right)$ can be found. Let $\lambda_{1}, \ldots, \lambda_{m} \geq 0$, then the set $C$ where the maximum $\Phi$ is attained is given by $C=\operatorname{conv}\left\{v_{1}, \ldots, v_{m}\right\}$. It means that for a (local or global) maximum point $x^{*}=V \mu$ of SCO $\mu_{m+1}, \ldots, \mu_{n}=0$. As $n-m$ elements of $\mu$ have a value of zero, $\mathrm{SCO}$ is now equivalent to

$$
\max _{C} \varphi(x)=\max \left\{\varphi(\hat{V} \hat{\mu}) \mid \sum_{1}^{m} \hat{\mu}_{j}=1, \hat{\mu} \geq 0\right\}
$$

where $\hat{\mu}$ and $\hat{V}$ now consist of the first $m$ elements of $\mu$ and $V$ respectively.

One can consider the problem as a lower dimensional covering verification. Unfortunately, we are not dealing with an equivalent SC problem. Besides the spheres $B_{1}, \ldots, B_{m}$ we have to deal with the cut $\hat{B}_{j}$ of spheres $B_{j}, j=m+1, \ldots, n$ with the plane of $C$. We will describe the plane and the intersection of the spheres that correspond to points that are not vertices of face $C$.

The plane can be described by

$$
\Gamma=\left\{x \in R^{d} \mid x=v_{1}+X y, y \in R^{m-1}\right\}
$$

where $X$ is again the matrix $X=\left[v_{2}-v_{1}, \ldots, v_{m}-v_{1}\right]$. The orthogonal projection of $v_{m+1}, \ldots, v_{n}$ on $\Gamma$ via projection matrix $P=X\left(X^{T} X\right)^{-1} X^{T}$ gives centre points $\hat{v}_{j}=$ $v_{1}+P\left(v_{j}-v_{1}\right)$. Notice that due to the projection, the power function value of a point $z \in \Gamma$ to one of the vertices $v_{j}$ is $\left\|z-v_{j}\right\|^{2}=\left\|z-\hat{v}_{j}\right\|^{2}+\left\|\hat{v}_{j}-v_{j}\right\|^{2}$. This means that the radii of the cuts of the spheres with plane $\Gamma$ are given by $\hat{r}_{j}^{2}=r_{j}^{2}-\left\|\hat{v}_{j}-v_{j}\right\|^{2}$. A negative value means that $B_{j}$ does not cut through plane $\Gamma$, so it does not have to be taken into account.

Problem (21) is lower dimensional than the original SC problem. Unfortunately, it is not easier as now we are dealing with a problem with $m$ vertices and $n-m$ other centres in the plane, not necessarily in $C$. We know that the maximum of $\varphi$ over $C$ can be found on the vertices of the power diagram in $C$ or at the intersection of the power diagram and the boundary of facet $C$. The power planes have the shape

$$
2\left(v_{i}-v_{j}\right)^{T} \hat{V} \hat{\mu}=\hat{r}_{i}^{2}-\hat{r}_{j}^{2}+\hat{v}_{i}^{T} v_{i}-v_{j}^{T} v_{j} \rightarrow a_{k}^{T} \hat{\mu}=b_{k}, \quad k=1, \ldots, K
$$


Fig. 7 Cross-cut of Example 2 with the plane through $v_{1}, v_{2}, v_{3}$

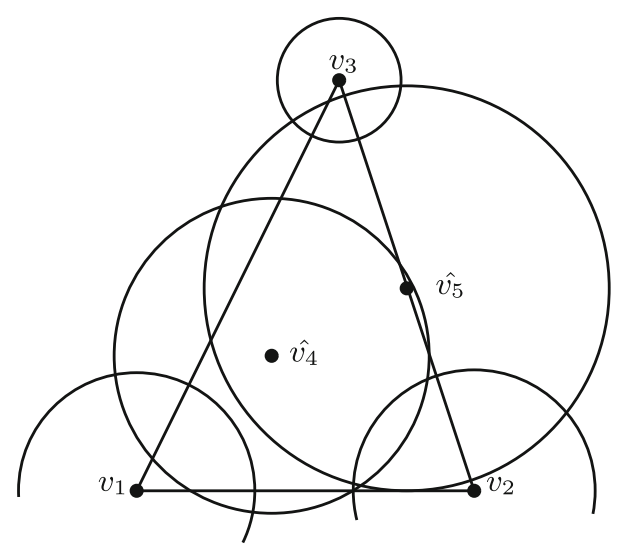

where not all $K=\frac{1}{2} n(n-1)$ power planes are of interest. One can argue that the power planes in between the vertices of $C$ are of less interest, as $x^{*}$ is not situated on those power planes and at the boundary of $C$, as one can improve the value of $\varphi$ by going to the interior.

Example 2 Consider the following instance of 5 spheres in 6-dimensional space:

$$
v_{1}=\left(\begin{array}{l}
0 \\
0 \\
0 \\
0 \\
0 \\
0
\end{array}\right), v_{2}=\left(\begin{array}{l}
5 \\
0 \\
0 \\
0 \\
0 \\
0
\end{array}\right), v_{3}=\left(\begin{array}{l}
3 \\
6 \\
0 \\
0 \\
0 \\
0
\end{array}\right), v_{4}=\left(\begin{array}{l}
2 \\
2 \\
0 \\
1 \\
1 \\
1
\end{array}\right), v_{5}=\left(\begin{array}{l}
4 \\
3 \\
1 \\
0 \\
2 \\
1
\end{array}\right)
$$

and $r_{1}^{2}=4, r_{2}^{2}=3, r_{3}^{2}=1, r_{4}^{2}=9, r_{5}^{2}=15$. Now solving system (18) and (19) gives $\lambda=(1.3422,0.8467,1.3444,-2.2333,-0.3000)^{T}$. The negative values in the vector mean $\theta=V \lambda=(2.6,2.7,-0.3,-2.233,-2.833,-2.533)^{T}$ is situated outside simplex $S$. It is at the same weighted power function value $\varphi(\theta)=29.57$ of $v_{1}, \ldots, v_{5}$, so $\theta$ is not covered. The positive values in the $\lambda$ vector indicate that we can look for the maximum of $\varphi$ on the convex hull $C$ of the first 3 vertices, as given by problem (21). Correspondingly, the cut of $B_{4}$ and $B_{5}$ can be found by projections $\hat{v}_{4}=(2,2,0,0,0,0)^{T}, \hat{v}_{5}=(4,3,0,0,0,0)^{T}$ and reduced radii $\hat{r}_{4}=6$ and $\hat{r}_{5}=9$. Figure 7 gives a $2 \mathrm{D}$ impression. The figure does not show us exactly whether $C$ is covered. However, using a solver one can verify that the global maximum $\Phi=-0.22$ can be found in the point $x^{*}=(3.333,0,0,0,0,0)^{T}$, and this means that $S$ is covered.

Global optimum $x^{*}$ is attained at one of the feasible basic solutions of a polyhedral set which describe the vertices of the power planes intersected with set $C$. To describe the polyhedral set we introduce free slack variables $z_{k}$ of the power planes and define set $H$ as

$$
H=\left\{\hat{\mu} \in R^{m}, z \in R^{K} \mid \sum_{1}^{m} \hat{\mu}_{j}=1, \hat{\mu} \geq 0, a_{k}^{T} \hat{\mu}-b_{k}=z_{k}, \quad k=1, \ldots, K\right\} .
$$

As $\varphi$ is piecewise convex it will have local optima at the feasible basic solutions of $H$, but one does not know which one corresponds to the global maximum $\Phi$. This shows that $\mathrm{SC}$ is a problem which is hard to solve. Mainly this will be the case for instances with a large number of vertices representing an irregular simplex where none of the spheres covers other vertices. 


\subsection{The regular case}

The question is whether the SC problem is easier to solve for regular simplices. The first check in the verification is of course to see whether one of the radii is big enough to cover the complete simplex. The next step is to generate the $\theta$ point and to check whether it is covered. If the latter is not the case, one should consider problem (21) on the lower dimensional plane $C$. The regular case reveals a specific property here. Due to the equal distance of a vertex $v_{j}, j=m+1, \ldots, n$ to all vertices $v_{i}, i=1, \ldots, m$, the projection $\hat{v}_{j}=P v_{j}$ on $C$ also gives an equal distance point. This means that all projected vertices $\hat{v}_{j}$ coincide at the centroid of $C$.

Example 3 Consider the following instance of 5 spheres in 5-dimensional space:

$$
v_{1}=\left(\begin{array}{l}
0 \\
0 \\
0 \\
0 \\
0
\end{array}\right), v_{2}=\left(\begin{array}{r}
-3 \\
3 \\
0 \\
0 \\
0
\end{array}\right), v_{3}=\left(\begin{array}{r}
-3 \\
0 \\
3 \\
0 \\
0
\end{array}\right), v_{4}=\left(\begin{array}{r}
-3 \\
0 \\
0 \\
3 \\
0
\end{array}\right), v_{5}=\left(\begin{array}{r}
-3 \\
0 \\
0 \\
0 \\
3
\end{array}\right)
$$

and $r_{1}^{2}=4, r_{2}^{2}=4, r_{3}^{2}=4, r_{4}^{2}=15, r_{5}^{2}=12$. The power function value between each pair of points is 18 .

Now solving system (18) and (19) gives $\lambda=(.41, .41, .41,-.2,-.0333)^{T}$. Point $\theta=$ $V \lambda=(-1.7667,1.2333,1.2333,-.6,-.1)^{T}$ is outside simplex $S$ at the same weighted power function value $\varphi(\theta)=2.533$ from $v_{1}, \ldots, v_{5}$, so $\theta$ is not covered. Projecting now via the $P$ matrix determined by $X=\left[v_{2}-v_{1}, v_{3}-v_{1}\right]$ gives the centroid of $C=\operatorname{con} v\left\{v_{1}, v_{2}, v_{3}\right\}$ : $\hat{v}_{4}=\hat{v}_{5}=(-2,1,1,0,0)^{T}$. In the reduced problem, only $\hat{r}_{4}^{2}=15-\left\|\hat{v}_{4}-v_{4}\right\|^{2}=$ $15-12=3$ is relevant, as $\hat{r}_{5}=0$. Due to the symmetry in this instance, the global maximum $\Phi=-1.2778$ is attained at several points on the boundary; vector $\hat{\mu}$ shows a permutation of the values $0,0.611$ and 0.389 .

\section{Conclusions}

The Simplex Cover (SC) problem to determine whether a given simplex in $d$ dimensional space is covered by $n$ spheres centered at its vertices has been investigated. It is shown that this problem is equivalent to solving a Global Optimization problem SCO. The following has been found.

- Depending on the instance, SCO may have a unique interior optimum $x^{*}$ which equals the so-called vertex point $\theta$ of a power diagram. A procedure is described to find this $\theta$ point.

- If the $\theta$ point is covered by the spheres, the simplex is covered, independently of $\theta$ being located in or outside the simplex.

- If SCO has a boundary optimum it may have local non-global optima.

- In the latter case, the $\theta$ point determines the face $C$ of the simplex where the global optimum points of SCO can be found. A global optimum point is a feasible basic solution of a polyhedral set which is determined by $C$ and so-called power planes.

- In the latter case, the SC problem appears to be equivalent to a problem of covering $C$ with more spheres than the ones centered at the vertices. 
- For a regular instance with all distances between vertices equal, the optimum point of $\mathrm{SCO}$ is either unique, or $\mathrm{SCO}$ is equivalent to the question of covering $C$ with the spheres at its vertices plus a sphere at its centroid.

As future research we are interested in developing efficient procedures (algorithms) which can be used as infeasibility tests in the context of the solution of blending problems by Branch-and-Bound approaches, where simplices appear as a logical partition set.

Open Access This article is distributed under the terms of the Creative Commons Attribution Noncommercial License which permits any noncommercial use, distribution, and reproduction in any medium, provided the original author(s) and source are credited.

\section{References}

1. Aurenhammer, F.: Voronoi diagrams - a survey of a fundamental geometric data structure. ACM Comput. Surv. 23(3), 345-405 (1991). doi:10.1145/116873.116880

2. Casado, L.G., Hendrix, E.M.T., García, I.: Infeasibility spheres for finding robust solutions of blending problems with quadratic constraints. J. Glob. Optim. 39(2), 215-236 (2007). doi:10.1007/ s10898-006-9072-6

3. Edelsbrunner, H.: The union of balls and its dual shape. In: SCG '93: Proceedings of the Ninth Annual Symposium on Computational Geometry, pp. 218-231, ACM, New York, USA (1993)

4. Gavrilova, M., Rokne, J.: An efficient algorithm for construction of the power diagram from the voronoi diagram in the plane. Int. J. Comput. Math. 61, 49-61 (1997) http://pages.cpsc.ucalgary.ca/ marina

5. Hendrix, E.M.T., Casado, L.G., García, I.: The semi-continuous quadratic mixture design problem: description and branch-and-bound approach. Eur. J. Oper. Res. 191(3), 803-815 (2008). doi:10.1016/j.ejor.2007. 01.056

6. Sadri, B.: Surface and medial axis topology through distance flows induced by discrete samples. PhD thesis, University of Illinois, Urbana, Illinois (2006). http://www.cs.duke.edu/ sadri 